\title{
Hybrid photonic system integration using thin glass platform technology (Erratum)
}

\author{
Henning Schröder, ${ }^{a, b}, *$ Julian Schwietering, ${ }^{b}$ Gunnar Böttger, ${ }^{a}$ and \\ Vanessa Zamora ${ }^{\text {a,b }}$
}

${ }^{\mathrm{a}}$ Fraunhofer Institute for Reliability and Microintegration (IZM), Berlin, Germany

${ }^{\mathrm{b}}$ Technical University of Berlin, Berlin, Germany

[DOI: 10.1117/1.JOM.1.4.049801]

This article [J. Optical Microsystems 1 (3), 033501 (2021) https://doi.org/10.1117/1.JOM.1 .3.033501] was originally published on 14 May 2021, with an incorrect reference list. The correct reference list was provided by the authors, and the paper was republished on 28 December 2021.

*Address all correspondence to Henning Schröder, henning.schroeder@izm.fraunhofer.de 